\title{
Optical Method to Determine Gait Parameters Using Position Sensitive Detector
}

\author{
Gu-In Jung*, Ji-Sun Kim*, Tae-Hee Lee*, Ju-Hyeon Choi*, Han-Byeol Oh*, A-Hee Kim*, \\ Bong-Jun Goh*, Jun-Sik Kim*, Eun-Suk Lee* and Jae-Hoon Jun ${ }^{\dagger}$
}

\begin{abstract}
This study suggests an optical method to measure cardinal of gait (step width, step length, and stride length) with position sensitive detector (PSD). The effect of reflector's shape (flat and cylinder) on the PSD output voltage was examined for the application of the suggested system to real situations with a curved shape reflector (e.g. shoes). Various mathematical models were evaluated to find the optimal equation for the distance measurement. Considering the effect of shape on detected signal, the inverse polynomial model was developed. The suggested method is simple to operate, low in cost, small in size, and can evaluate gait parameters in real time. This method is expected to be useful in the field of rehabilitation and sport science
\end{abstract}

Keywords: Position sensitive detector, Gait, Walking, Gait parameters, Rehabilitation

\section{Introduction}

For the rehabilitation process, measurements of the temporal and spatial features of gait are very important for monitoring functional recovery [1]. Quantitative gait analysis is required to evaluate the effects of various rehabilitation treatments designed for improving patient's gait disability [2-5].

Many methods have been studied to evaluate walking pattern. Measuring foot prints from patient's painted feet was used to evaluate walking pattern. This method was simple but had limitations in space and accuracy. Walking kinematics can be evaluated with accelerometer but it can be shock-sensitive and has not enough accuracy due to double integration of acceleration for the derivation of displacement [6-10].

The 3-D motion capture system with multiple IR cameras is widely used. However, the equipment is very expensive and can only be used in a specialized motion laboratory. To process data, specialists are required and the data processing time is relatively long. The system requires calibration in each test set and long preparation time. Sometimes patients claim inconvenience in attaching reflection makers on their body [11-12].

Force plate method requires multiple force sensors to measure walking cycle within limited plate area [13]. This method has difficulty in evaluation walking pattern of

$\dagger$ Corresponding Author : Dept. of Biomedical Engineering, BK21 Plus Research Institute of Biomedical Engineering, College of Biomedical \& Health Science, Konkuk University, Chungju, Republic of Korea. (jjun81@kku.ac.kr)

* Dept. of Biomedical Engineering, BK21 Plus Research Institute of Biomedical Engineering, College of Biomedical \& Health Science, Konkuk University, Chungju, Republic of Korea. (guin83@ konkuk.ac.kr, \{kimji0330, t-a-e-h-e-e, wngus829, xhs1852, aheek, bey1212, nattee,eunsuk8097\}@nate.com)

Received: October 10, 2013; Accepted: May 15, 2015

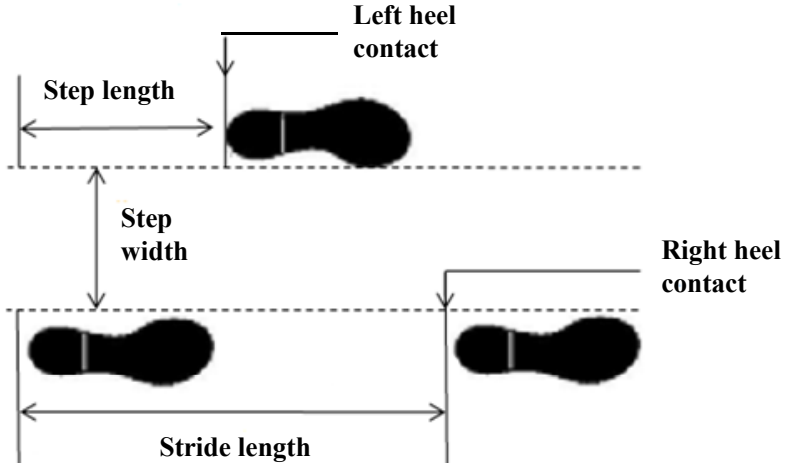

Fig. 1. Definition of gait parameters

patients with paralysis. Foot switch method can be used for detection of foot contacts on the ground. This method is useful for normal gait analysis but not suitable for pathological gait because of abnormal contact patterns such as loss of heel contact [14-17].

The purpose of this study is to suggest alternative method for the identification of gait feature which would be convenient, inexpensive, and operating in real time. According to the principle of triangulation method, position sensitive detector (PSD) converts detect reflected light into distance-related voltage. After mathematical modeling of PSD output, we can obtain step width, step length, and stride length. Our final objective is to monitor gait parameters without time and space limitations using wireless module.

\section{Materials and Methods}

\subsection{Gait features}

Walking patterns can be described by gait phase, gait 
cycle, step length, and stride length. Stride length indicates the distance between two sequential initial contacts of the identical foot (e.g., right to right), step length indicates the distance between initial contact of one foot and the other foot (e.g., right to left), step width indicates the mediolateral distance between the locations of sequential initial contacts by two feet (see Fig. 1) [18].

\subsection{Position sensitive detector (PSD)}

Detection of distance in PSD is based on the principle of triangulation method [19-20]. PSD is not be affected by the color of reflector, while most optical sensors show change in the light intensity when reflected by different colors. PSD detects reflected light which induces electric current. The electric current ratio is determined by the hitting point of reflected light on the sensing area of light detector which converts the current ratio into voltage as an output signal.

Fig. 2 shows the principle of triangulation method in PSD sensor. Eq. (1) describes the relationship among lengths $\left(\mathrm{L}_{1}, \mathrm{~L}_{2}, \mathrm{X}_{1}\right.$, and $\left.\mathrm{X}_{2}\right)$. The angle of incident beam can be changed by the ratios $\mathrm{L}_{1} / \mathrm{L}_{2}$ and $\mathrm{X}_{1} / \mathrm{X}_{2}$. PSD sensor (GP2Y0A21YK0F, sharp, Japan) module is composed of light emitter (IR diode), lenses, and light detector. When the distance between sensor and reflectors is long, the incident beam hit the right part of the sensing area of light detector (see solid line). Likewise, when the distance between sensor and reflector is short, the incident beam hit the left part of the sensing area of light detector (see dashed line).

$$
L_{1}: X_{1}=L_{2}: X_{2}
$$

where $\mathrm{L}_{1}$ is the distance between lens 1 and reflecting object, $\mathrm{L}_{2}$ is the distance between lens 2 and light detector (fixed), $X_{1}$ is the distance between lens1 and lens2 (fixed), $\mathrm{X}_{2}$ is the distance between normal point to lens 2 and the hitting point of incident light in the sensing area of light

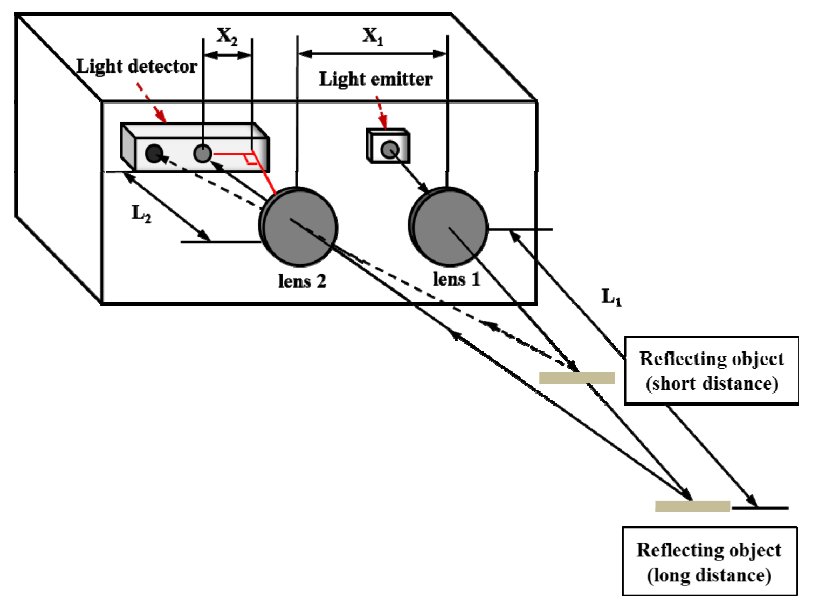

Fig. 2. PSD sensor's signal detection principles

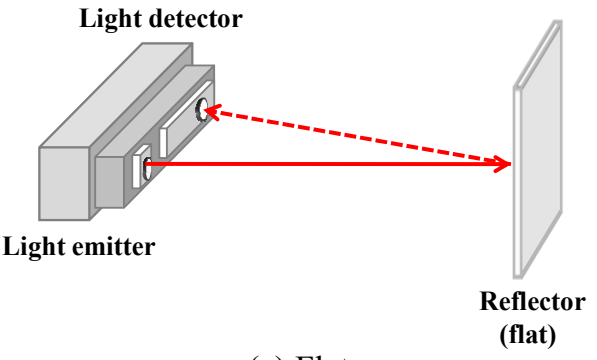

(a) Flat

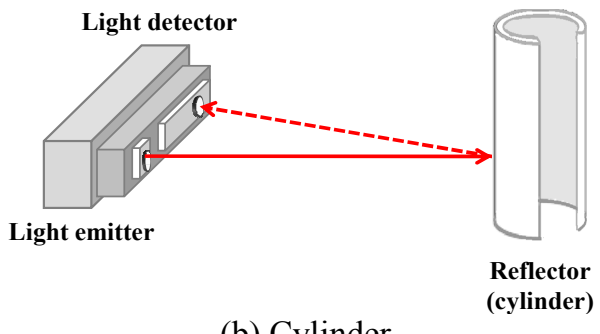

(b) Cylinder

Fig. 3. Setup for reflector's shape

detector.

The distance measuring range is $10-80 \mathrm{~cm}$. It provides an analog output voltage that is correlated to the distance. Its supply voltage can be from 4.5 to $5.5 \mathrm{~V}$ and typical average dissipation current can be from 30 to $40 \mathrm{~mA}$.

\subsection{The effect of reflector's shape}

For the application of PSD to real situations, curved shape reflector must be considered. The effect of reflector's shape on PSD output voltage was investigated with specially designed setups. Reflecting objects such as ankle and shoes are not perfectly straight. They usually have curved shapes. Experimental setup was designed to test the effect of reflector's shape (see Fig. 3). The diameter of cylindrical reflector was $8 \mathrm{~cm}$ which was similar to shoe's heel shape. The PSD sensor is fixed on an optical table. The distance between the sensor and the reflectors was changed by an optical rail (PRL-24, Newport, Irvine, CA) with a resolution of $0.005 \mathrm{~cm}$.

The obtained results could be useful for realistic applications on shoes or ankles.

\subsection{Data acquisition and calibration}

Output signals from PSD sensor were collected using DAQ board (NI USB-6008, National Instrument, Austin, Texas) in digital form, and saved in a computer with LabVIEW (NI LabVIEW 8.0, National Instruments, Austin, Texas). Modeling of the experimental data was done by using Matlab (R2008a, Mathworks, Portola Valley, California).

Polynomial equations ( $1^{\text {st }}$ to $5^{\text {th }}$ order), exponential function, power function, logarithmic function, and fractional function were tested for calibration. Forward and 
inverse mathematical models were selected to obtain the distance. First, the relationship between PSD output and distance was mathematically modeled. Next, the inverse relationship was used to obtain the distance.

The inverse model is possible when the functions show monotonic pattern (increase or decrease). Eqs. (2)-(6) show inverse models with polynomial equations ( $1^{\text {st }}$ to $5^{\text {th }}$ order $)$, exponential function, power function, logarithmic function, and fractional function.

$$
\begin{gathered}
x=a_{0} y^{n}+a_{1} y^{n}+a_{2} y^{n}+\ldots+a_{n} y^{0} \\
x=b_{0} \exp \left(b_{1} y\right) \\
x=c_{0} y^{c_{1}} \\
x=d_{0} \log (y)+d_{1} \\
x=1 /\left(e_{0} y+e_{1}\right)
\end{gathered}
$$

where $a_{0} \sim a_{n}$ are coefficients of polynomial equation, $b_{0}$ $\sim \mathrm{b}_{1}$ are coefficients of exponential function, $\mathrm{c}_{0} \sim \mathrm{c}_{1}$ are coefficients of power function, $\mathrm{d}_{0} \sim \mathrm{d}_{1}$ are coefficients of logarithmic function, $\mathrm{e}_{0} \sim \mathrm{e}_{1}$ are coefficients of fractional function, $\mathrm{x}$ is the distance $[\mathrm{cm}]$, and $\mathrm{y}$ is the PSD output voltage [V].

\subsection{Measurements of gait parameters}

Fig. 4 shows schematic of the calculation of gait parameters. Two PSD sensors were used to measure the step width and the cross distance between toe and heel. The role of PSD 1 and PSD 2 were to obtain the cross distance between toe and heel $\left(\mathrm{S}_{1}\right)$ and step width $\left(\mathrm{S}_{2}\right)$, respectively. After, applying Eqns. (7)-(9), step width, step length, and stride length can be calculated and monitored with LabVIEW in real time

Four healthy young adults (two females and two males, mean age $=25.75 \pm 3.5$ years old) participated in the study. The gait parameters such as step width, step length, and stride length were evaluated in static positions. To test the possibility of PSD sensor for gait analysis, human subjects wore the shoes attached with PSD sensor as shown in Fig. 4.

$$
\begin{gathered}
S_{3}=\left(S_{1}^{2}-S_{2}^{2}\right)^{1 / 2} \\
\text { Step length }=S_{3}+\mathrm{fl} \\
\text { Stride length }=2\left(S_{3}+\mathrm{fl}\right)
\end{gathered}
$$

where $S_{1}$ is the cross distance between toe and heel [cm], $\mathrm{S}_{2}$ is the step width [cm], $\mathrm{S}_{3}$ is the normal distance between toe of one foot and heel of the other foot $[\mathrm{cm}]$, and $\mathrm{fl}$ is the foot length $[\mathrm{cm}]$.

To decrease the deviation of measuring distance by moving direction of the reflective object, we considered to set the sensor that the moving direction of the reflector and the line between emitter center and detector center are vertical. The sensors were mounted vertically and the shoes

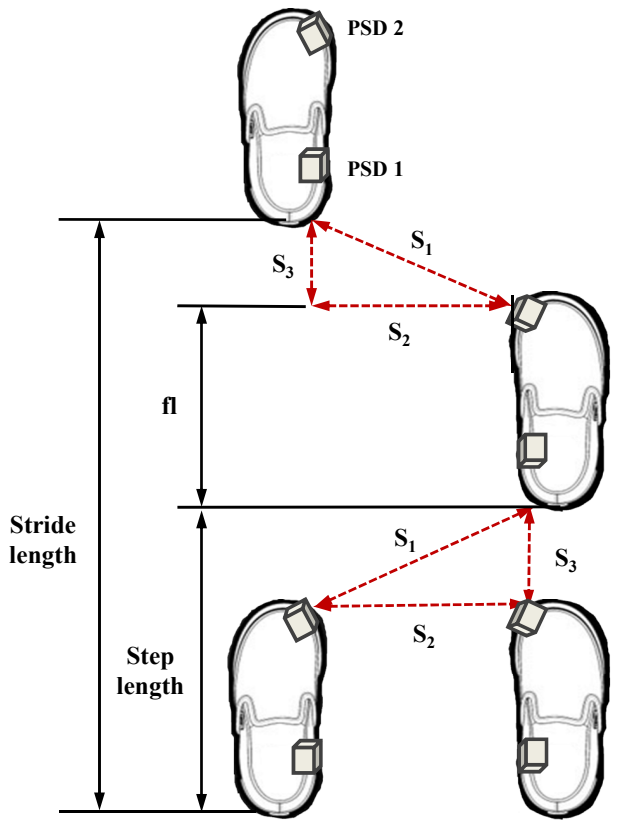

Fig. 4. Schematic for the calculation of gait parameters

moved horizontally.

\section{Results and Discussion}

\subsection{The effect of reflector's shape}

Fig. 5 shows the effect of reflector's shape on PSD output. Flat and cylinder reflectors show some differences in short distance up to $15 \mathrm{~cm}$, while differences are negligible in long distance. Various models were applied to find the optimal function to get distance.

Table 1 shows coefficients of determination $\left(\mathrm{R}^{2}\right)$ and root-mean-square error (RMSE) with polynomial equations $\left(1^{\text {st }}\right.$ to $5^{\text {th }}$ order$)$, exponential function, power function, logarithmic function, and fractional function. The results shows $5^{\text {th }}$ order polynomial equation has the optimal $\mathrm{R}^{2}$ and the minimum RMSE for both flat and cylinder reflectors. Thus $5^{\text {th }}$ order polynomial function was used for inverse solution model to obtain distance.

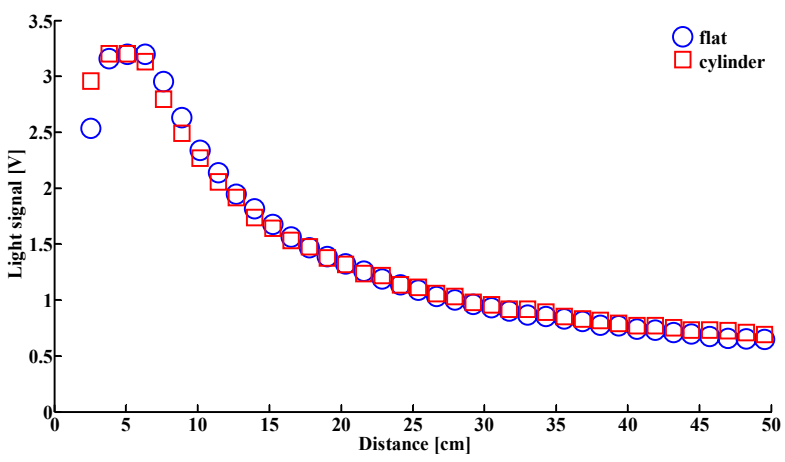

Fig. 5. Effect of reflector's shape 
Table 1. Modeling results ( $\mathrm{R}^{2}$ and RMSE)

\begin{tabular}{c|c|c|c|c}
\hline \multirow{2}{*}{ Equation } & \multicolumn{2}{|c|}{$\mathrm{R}^{2}$} & \multicolumn{2}{c}{ RMSE } \\
\cline { 2 - 5 } & flat & cylinder & flat & cylinder \\
\hline $1^{\text {st }}$ & 0.8169 & 0.8119 & 0.2620 & 0.2430 \\
\hline $2^{\text {nd }}$ & 0.9690 & 0.9689 & 0.1096 & 0.1003 \\
\hline $3^{\text {th }}$ & 0.9945 & 0.9942 & 0.0468 & 0.0441 \\
\hline $4^{\text {th }}$ & 0.9991 & 0.9988 & 0.0197 & 0.0204 \\
\hline $5^{\text {th }}$ & 0.9998 & 0.9995 & 0.0007 & 0.0126 \\
\hline exponential & 0.9416 & 0.9316 & 0.1479 & 0.1466 \\
\hline power & 0.9980 & 0.9992 & 0.0009 & 0.0156 \\
\hline logarithmic & 0.9574 & 0.9548 & 0.1265 & 0.1192 \\
\hline fractional & 0.9984 & 0.9949 & 0.0242 & 0.0401 \\
\hline & \multicolumn{3}{|c}{}
\end{tabular}

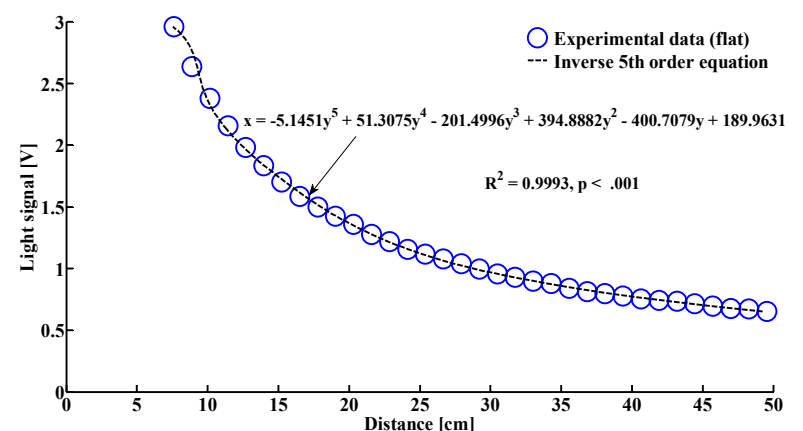

(a) Flat

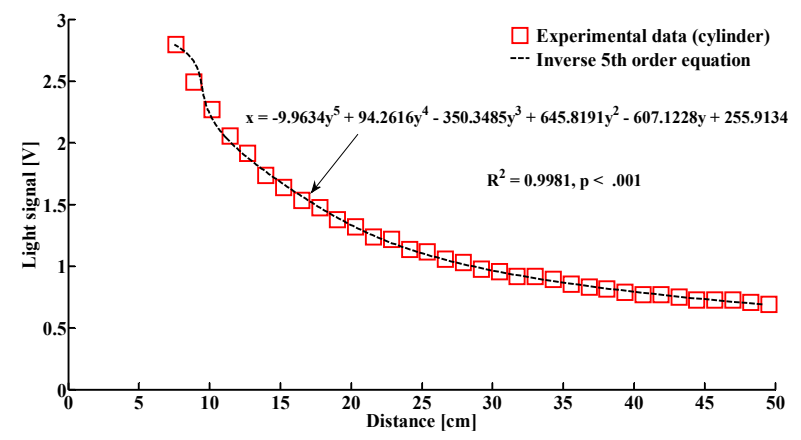

(b) Cylinder

Fig. 6. Inverse of $5^{\text {th }}$ order polynomial

Fig. 6 shows calibration results with inverse $5^{\text {th }}$ order polynomial equation for flat and cylinder reflectors. Only monotonically decreasing region was used to get inverse $5^{\text {th }}$ order equation. Inverse of $5^{\text {th }}$ order equation had coefficients of determination of flat $\left(\mathrm{R}^{2}=0.993\right.$ with $\mathrm{p}<.001)$ and cylinder reflectors $\left(\mathrm{R}^{2}=0.9981\right.$ with $\left.\mathrm{p}<.001\right)$.

\subsection{Measurements of gait parameters}

The PSD sensors were attached on a pair of shoes and experiments were performed to find the optimal mathematical model for the use in gait analysis. In this experiment, PSD sensors were attached on one shoe and the other shoe was used as reflector.

Fig. 7 shows calibration results with inverse $5^{\text {th }}$ order polynomial equation of shoe model $\left(\mathrm{R}^{2}=0.991\right.$ with $\mathrm{p}<.001)$.

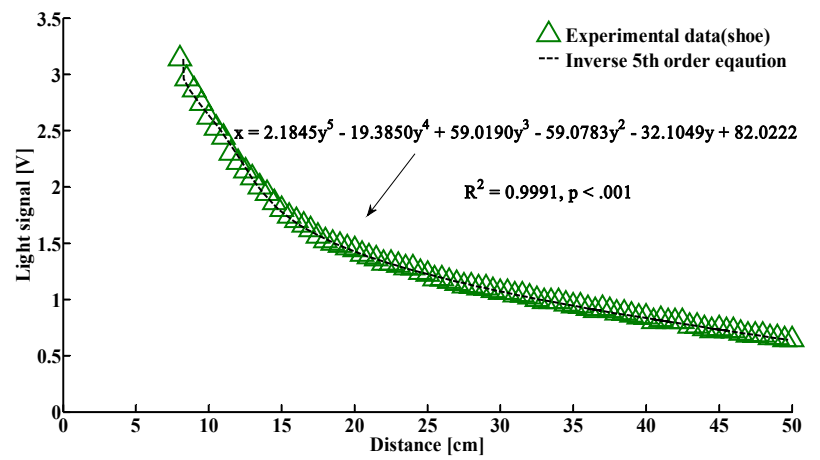

Fig. 7. Inverse of $5^{\text {th }}$ order polynomial for shoe model

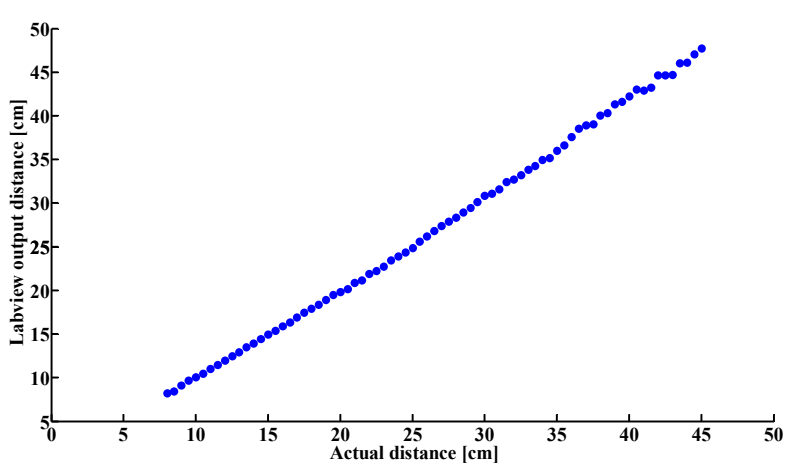

Fig. 8. Actual distance versus calculated distance with shoe model

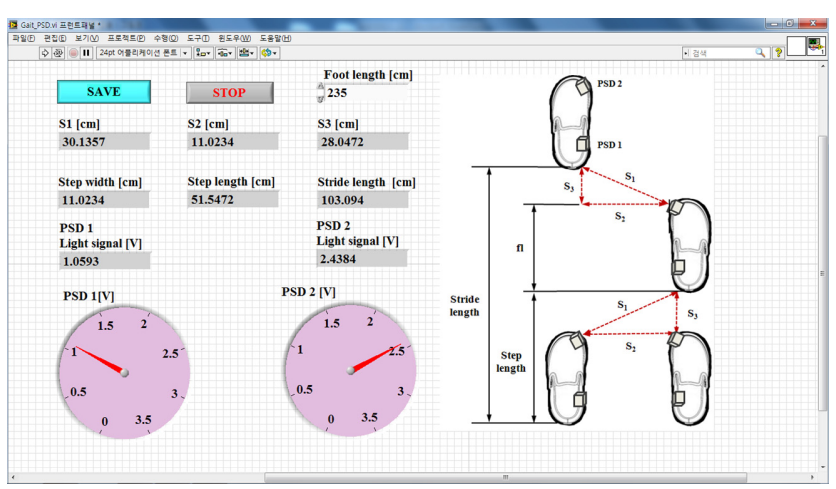

Fig. 9. Example of LabVIEW display

Fig. 8 shows relationship between the actual distance and LabVIEW output distance. The result showed actual distance was correlated well with calculated LabVIEW output distance. Experimental results showed that the RMSE was $\pm 0.3185 \mathrm{~cm}$.

Fig. 9 shows LabVIEW display to get the distance from the inverse of $5^{\text {th }}$ order polynomial equation in real time. For measurements with human subjects wearing the sensor attached shoes, step length and stride length were calculated using gait parameters described in Eqns. (7)-(9) and Fig. 4.

Fig. 10 shows the gait parameters such as step width, step length, and stride length in four subjects (two female and two male) in static positions. To test the possibility of 


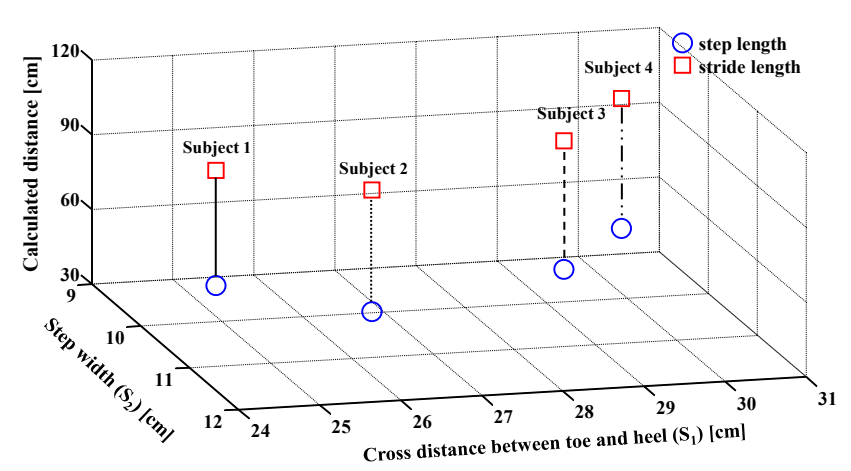

Fig. 10. Measurement of gait parameters

PSD sensor for gait analysis, human subject wore the shoes attached with PSD sensor as shown in Fig. 4. By measuring the cross distance between toe and heel $\left(\mathrm{S}_{1}\right)$ and step width $\left(\mathrm{S}_{2}\right)$, step length and stride length could be obtained using Eqns. (7)-(9). The relationship between the reference distance and the calculated distance output was evaluated. Experimental results with four subjects showed that the inverse $5^{\text {th }}$ order equation had RMSEs of gait parameters such as step width, step length, and stride length were $\pm 0.0135 \mathrm{~cm}, \pm 0.7792 \mathrm{~cm}$, and $\pm 1.5584 \mathrm{~cm}$, respectively. Other models such as power function and fractional function (see Table 1) were also tested for possible practical use. Inverse power function had RMSEs of gait parameters such as step width, step length, and stride length were $\pm 0.1654 \mathrm{~cm}, \pm 0.8419 \mathrm{~cm}$, and \pm 1.6839 $\mathrm{cm}$, respectively. Also, inverse power function had RMSEs of gait parameters such as step width, step length, and stride length were $\pm 0.2436 \mathrm{~cm}, \pm 1.0398 \mathrm{~cm}$, and \pm 2.0796 $\mathrm{cm}$, respectively. Both power function and fractional function were good for possible use; however, the inverse $5^{\text {th }}$ order equation was the best model with the smallest RMSEs of gait parameters. Measured values were correlated with reference distances in test range.

The attached angles in the range of $\pm 4^{\circ}$ deviation were tested and the RMSE of $S_{1}$ was $\pm 0.4449 \mathrm{~cm}$. As results, the gait parameter errors such as the RMSEs of step width, step length, and stride length were $\pm 0.0135 \mathrm{~cm}, \pm 0.7792$ $\mathrm{cm}$, and $\pm 1.5584 \mathrm{~cm}$, respectively. The step width $\left(\mathrm{S}_{2}\right)$ is independent of $S_{1}$ while the step length and stride length are dependent on $S_{1}$. The errors of the step length and stride length can be increased as the error of $\mathrm{S}_{1}$ is increased.

Even though the optical method has limitation in accuracy, the suggested technique can be used conveniently using a remote module without space limitation and in real time using LabVIEW display. We suggested a new optical method to obtain gait parameters. In future study, we will reduce the $S_{1}$ error with multiple sensors and the better sensor design.

Our results are not perfectly accurate at this stage and have difficulty in collecting data during continuous dynamic motion. Wireless module should be added in future study for dynamic motion without limitations of testing space.

The suggested optical sensor is more durable than the accelerometer-based sensor. Even though the camera-based system has the high precision of human motion, the camera equipment is very expensive and can only be used in a motion laboratory with specialists performing data analysis. The proposed sensor can be used without space limitation and in real time with the LabVIEW program. Thus, the proposed sensor has advantages that other commercial products cannot offer.

At least, the results of this study imply that optical method using PSD sensor has high possibility to perform gait analysis. Further study and better design should be done to get data during dynamic motion. We may use multiple PSD sensors with ultrasound sensors for better accuracy. Wireless module to transfer the sensor output signal to PC should be done for monitoring gait parameters which may reduce time and space limitations. By differentiation of distance, further information about walking speed and acceleration may be obtained. The results can be applied in the field of rehabilitation and sport science.

\section{Conclusion}

In this study, we developed an optical method to measure step width, step length, and stride length using PSD sensors. This sensor is based on the principle of triangulation method. While most optical sensors show color dependency, PSD sensor is not affected by reflector's color. Thus, we can collect distance data without color dependency and this is a very strong benefit as optical sensors.

The effect of reflector's shape was investigated with flat and cylinder reflectors to get accurate distance information. Various mathematical models such as polynomial equations, exponential function, power function, logarithmic function, and fractional function were evaluated to find the optimal equation. With $5^{\text {th }}$ order polynomial equation, we obtained the best $\mathrm{R}^{2}$ and the minimum RMSE. In this study, PSD sensors were attached on one shoe and the other shoe was used as reflector. The result showed that actual distance was correlated well with calculated LabVIEW output distance. The measured gait parameters with human subjects imply that our optical method can be used for gait analysis. This study can be applied in the field of rehabilitation and sport science.

\section{Acknowledgements}

This research was supported by Basic Science Research Program through the National Research Foundation of Korea (NRF) funded by the Ministry of Education, Science and Technology (No. 2010-0023158) and the Pioneer Research Center Program through the National Research Foundation of Korea funded by the Ministry of Science, ICT \& Future planning (No. 2011-0027920). 


\section{References}

[1] Masaki Sekin, Yoshiyuki Abe, Mitsuyoshi Sekimoto, Yuji Higashi, Toshiro Fujimoto, Toshiyo Tamura, and Yasuhiro Fukui, "Assessment of gait parameter in hemiplegic patients by accelerometry," in Proc. Annu. Int. Conf. IEEE Eng. Med. Biol. Soc., vol. 3, pp. 1879-1882, July 2000.

[2] Ju-Won Lee and Gun-Ki Lee, “Adaptive postural control for trans-femoral prostheses based on neural networks and EMG signals," Int. J. Precis. Eng. Manuf., vol. 6, no. 3, pp. 37-44, July 2005.

[3] Gyoosuk Kim, Shinill Kang, Sungjae Kang, Jeicheong Ryu, Museoung Mun, and Kyunghoon Kim, "Unlockable knee joint mechanism for powered gait orthosis," Int. J. Precis. Eng. Manuf., vol. 10, no. 3, pp. 83-89, July 2009.

[4] Se-Jin Kong, Chul-Seung Kim, Ki-Wook Moon, Gwang-Moon Eom, Gye-Rae Tack, Kyeong-Seop Kim, Jeong-Whan Lee, and Young-Hee Lee, "GaitEvent Detection using an Accelerometer for the Paralyzed Patients," Trans. KIEE, vol. 56, no. 5, pp. 990-992, May 2007.

[5] Ki-Wook Moon, Chul-Seung Kim, Jae-Ho Lee, YuRi Kwon, Dong-Won Kang, Gon Khang, Yo-Han Kim, and Gwang-Moon Eom, "Development of Joint Angle Measurement System for the Feedback Control in FES Locomotion," Trans. KIEE, vol. 58, no. 1, pp. 203-209, Jan. 2009.

[6] Henk J. Luinge, and Peter H. Veltink, "Inclination measurement of human movement using a 3-D accelerometer with autocalibration," IEEE Trans. Neural Syst. Rehabil. Eng., vol. 12, no. 1, pp. 112121, Mar. 2004.

[7] Ruth E. Mayagoitia, Anand V. Nene, and Peter H. Veltink, "Accelerometer and rate gyroscope measurement of kinematics: an inexpensive alternate to optical motion analysis systems," J. Biom., vol. 35, no. 4, pp. 537-542, Apr. 2002.

[8] J.R.W. Morris, "Accelerometry-A technique for the measurement of human body movement," J. Biom., vol. 6, no. 6, pp.729-736, Nov. 1973.

[9] Dietrich Marcuse, "Curvature lose formula for optical fibers," J. Opt. Soc. Am., vol. 66, no. 3, pp. 216-220, 1976.

[10] A.L. Evans, G. Duncan, and W. Gilchrist, "Recording accelerations in body movements," MED. Biol. Eng. Comput., vol. 29, no. 1, pp. 102-104, Jan. 1991.

[11] Auvinet Bernard, Chaleil Denis, and Barrey Eric, "Accelerometric gait analysis for use in hospital outpatients," Rev. Rhum. Engl. Ed., vol. 66, no. 7-9, pp. 389-397, July-Sep. 1999.

[12] Michael W. Whittle, "Calibration and performance of a 3-dimensional television system for kinematic analysis," J. Biom., vol. 15, no. 3, pp. 185-196, 1982.

[13] Sun Woo Park, Ryang Hee Sohn, Ki Hong Ryu, and
Young Ho Kim, "Comparison of motion sensor system for gait phase detection," J. Korean Sco. Precis. Eng., vol. 27, no. 2, pp. 145-152, Feb. 2010.

[14] Jeffrey M. Hausdorf, Zvi Ladin, and d Jeanne Y. We, "Footswitch system for measurement of the temporal parameters of gait," J. Biom., vol. 28, no. 3, pp. 347351, Mar. 1995.

[15] Seung Chan Ann, Sung Jae Hwang, Sung Jae Kang, and Young Ho Kim, "Development and evaluation of a new gait phase detection system using FSR sensors and a gyrosensor," J. Korean Sco. Precis. Eng., vol. 21, no. 10, pp. 196-203, Oct. 2004.

[16] Brandell Bruce R., "Development of a universal control unit for functional electrical stimulation (FES)," Am. J. Phys. Med., vol. 61, no. 6, pp. 279301, Dec. 1982.

[17] Se-Jun Kong, Gwang-Moon Eom, Chul-Seung Kim, and Kwan-Yong Park, "Portable Gait-Event Detection System for FES Locomotion," Trans. KIEE, vol. 55D, no. 5, pp. 248-253, May 2006.

[18] Perry Jacquelin, "Gait analysis, Normal and pathological function," Slack, Thorofare, NJ, 1992.

[19] Q B Tong, Z L Ding, J C Chen, L L Ai, and F Yuan, "The research of screw thread parameter measurement based on position sensitive detector and laser," J. Phys.: Conf. Ser., vol. 48, pp. 561-565, 2006.

[20] Gu-In Jung, Ji-Sun Kim, Dong-Hun Hur, HwanDong Yu, Sung-Hwan Lim, Ju-Hyeon Choi, JeongHwan Lee, Gwang-Moon Eom, and Jae-Hoon Jun, "Angular Displacement Measurement Using Optical Sensor," Trans. KIEE, vol. 60, no. 10, pp. 1959-1965, Oct. 2011.

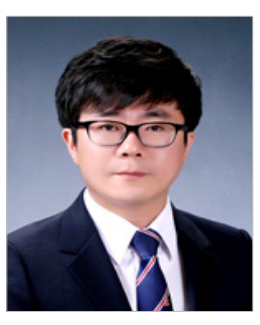

Gu-In Jung He received his B.S., M.S., and Ph.D. degrees in Biomedical Engineering from Konkuk University, Chungju, Korea, in 2008, 2010, and 2015 , respectively. At present, he is an advanced researcher in the Laser Application Center at Kyungpook National University.

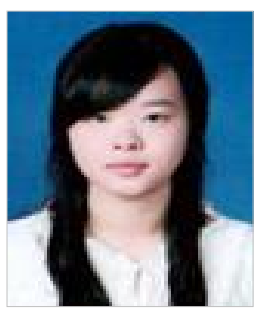

Ji-Sun Kim She received the B.S. degree and M.S. degree in Biomedical Engineering from Konkuk University (KKU), Chungju, South Korea, in 2009 and 2011. She is currently working toward the Ph.D. degree in Biomedical Engineering with the college of Biomedical \& Health Science, KonKuk University (KKU). Her research interests include laser tissue interaction and color detection. 


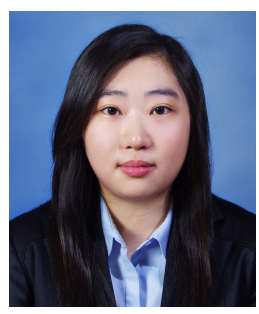

Tae-Hee Lee She received the B.S. degree and M.S. degree in Biomedical Engineering from Konkuk University (KKU), Chungju, South Korea, in 2011 and 2015. Her research interests include color distinguishing study and characterization of laser tissue interacttions.

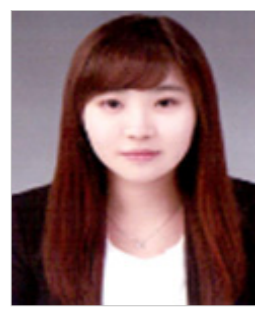

Ju-Hyeon Choi She received the B.S. degree and M.S. degree in Biomedical Engineering from Konkuk University (KKU), Chungju, South Korea, in 2013 and 2015. Her research interests include estimation of the light intensity by using LED sensory system, studies on interaction mechanism of laser.

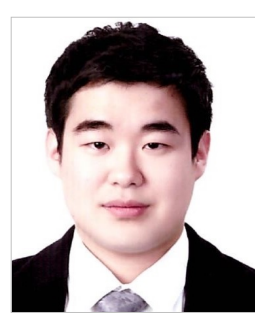

Han-Byeol Oh He received the B.S. degree in Department of Biomedical Engineering from Konkuk University (KKU), Chungju, South Korea, in 2014. $\mathrm{He}$ is currently working toward the M.S. degree in Department of Biomedical Engineering with the College of Biomedical and Health Science, KonKuk University (KKU). His research interests include simulation for optic, application of optical sensor, and studies on laser.

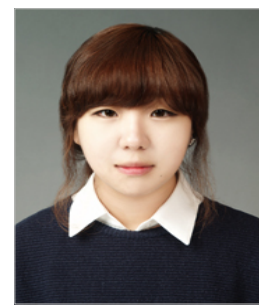

A-Hee Kim She received the B.S. degree in Department of Biomedical Engineering from Konkuk University (KKU), Chungju, South Korea, in 2014. She is currently working toward the M.S. degree in Department of Biomedical Engineering with the College of Biomedical and Health Science, KonKuk University (KKU). Her research interests include angle measurement design using optical fiber, studies on laser.

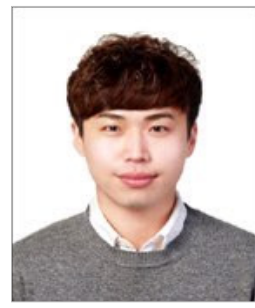

Bong-Jun Goh He received the B.S. degree in Department of Biomedical Engineering from Konkuk University (KKU), Chungju, South Korea, in 2015 $\mathrm{He}$ is currently working toward the M.S. degree in Department of Biomedical Engineering with the College of Biomedical and Health Science, KonKuk University (KKU). His research interests include application of optical sensor, and studies on laser.

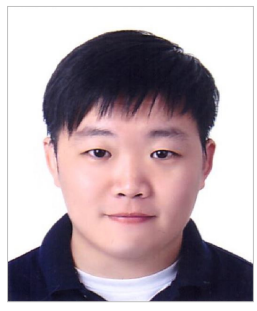

Jun-Sik Kim $\mathrm{He}$ received the B.S. degree in Department of Biomedical Engineering from Konkuk University (KKU), Chungju, South Korea, in 2015. $\mathrm{He}$ is currently working toward the M.S. degree in Department of Biomedical Engineering with the College of Biomedical and Health Science, KonKuk University (KKU). His research interests include angle measurement design using optical fiber, studies on laser.

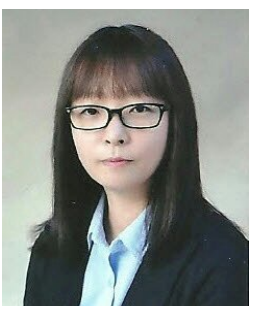

Eun-Suk Lee She received the B.S. degree in Department of Biomedical Engineering from Konkuk University (KKU), Chungju, South Korea, in 2015. She is currently working toward the M.S. degree in Department of Biomedical Engineering with the College of Biomedical and Health Science, KonKuk University (KKU). Her research interests studies on laser and color detection.

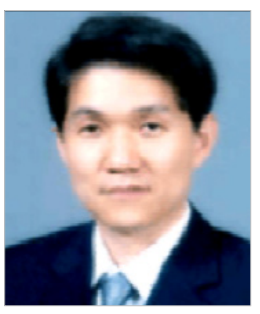

Jae-Hoon Jun He received the B.S. degree in Chemical Engineering from Korea University, Seoul, KOREA, the M.S. degree in Chemical Engineering and the Ph.D. degree in Biomedical Engineering from Texas A\&M University, College Station, TX, USA respectively. He was a Research Associate in Biomedical Engineering at Virginia Commonwealth University-Medical College of Virginia campus, Richmond, VA. He is currently a Professor in Biomedical Engineering at Konkuk University, Chungju, South Korea. His current research interests include optical sensing, photothermal therapy, tissue characterization, biomechanics, and T-ray imaging. 